\title{
Acetylcholine Facilitates Recovery of Episodic Memory after Brain Damage
}

\author{
Paula L. Croxson, ${ }^{1,2}$ Philip G. F. Browning, ${ }^{1,2}$ David Gaffan, ${ }^{2}$ and Mark G. Baxter ${ }^{1,2}$ \\ ${ }^{1}$ Glickenhaus Laboratory of Neuropsychology, Friedman Brain Institute, and Department of Neuroscience, Mount Sinai School of Medicine, New York, New \\ York 10029, and 2Department of Experimental Psychology, Oxford University, South Parks Road, Oxford OX1 3UD, United Kingdom
}

Episodic memory depends on a network of interconnected brain structures including the inferior temporal cortex, hippocampus, fornix, and mammillary bodies. We have previously shown that a moderate episodic memory impairment in monkeys with transection of the fornix is exacerbated by prior depletion of acetylcholine from inferotemporal cortex, despite the fact that depletion of acetylcholine from inferotemporal cortex on its own has no effect on episodic memory. Here we show that this effect occurs because inferotemporal acetylcholine facilitates recovery of function following structural damage within the neural circuit for episodic memory. Episodic memory impairment caused by lesions of the mammillary bodies, like fornix transection, was exacerbated by prior removal of temporal cortical acetylcholine. However, removing temporal cortical acetylcholine after the lesion of the fornix or mammillary bodies did not increase the severity of the impairment. This lesion order effect suggests that acetylcholine within the inferior temporal cortex ordinarily facilitates functional recovery after structural lesions that impair episodic memory. In the absence of acetylcholine innervation to inferotemporal cortex, this recovery is impaired and the amnesia caused by the structural lesion is more severe. These results suggest that humans with loss of cortical acetylcholine function, for example in Alzheimer's disease, may be less able to adapt to memory impairments caused by structural neuronal damage to areas in the network important for episodic memory.

\section{Introduction}

The neurochemical acetylcholine has been implicated in a range of cognitive processes, including episodic memory, which is impaired in Alzheimer's disease, in which there is a decrease in acetylcholine innervation (for review, see Bartus, 2000). However, systemic administration of acetylcholinesterase inhibitors produces only small acute improvements in memory (Sahakian et al., 1993). Despite the lack of evidence that procholinergic therapies in Alzheimer's disease lead to lasting improvements in memory, they do slow cognitive decline (Small et al., 2005).

One way to explain the lack of improvement in memory but a general preservation of cognitive decline in patients treated with cholinesterase inhibitors is that acetylcholine may have a role in boosting attentional performance or general cortical function (Parikh and Sarter, 2008). However, another explanation is that the loss of cortical acetylcholine in Alzheimer's disease may interact with the effects of direct damage to structures involved in memory. Neuropsychological, imaging, and animal lesion work has converged on an extended circuitry for episodic memory including (1) the inferior temporal (inferotemporal) cortex, in-

Received June 21, 2012; revised Aug. 2, 2012; accepted Aug. 15, 2012.

Author contributions: D.G. and M.G.B. designed research; P.L.C., P.G.F.B., D.G., and M.G.B. performed research; P.L.C., P.G.F.B., and M.G.B. analyzed data; P.L.C. wrote the paper.

This research was supported by a grant from the Wellcome Trust (to M.G.B.). We thank C. Bergmann, G. Daubney, D. Kyriazis, K. Murphy, C. Rae, and C. Wilson for technical assistance. We are grateful to P. H. Rudebeck, C. Alberini, and the Mount Sinai Postdoctoral Writing Group for helpful comments on the manuscript.

The authors declare no financial conflicts of interest.

Correspondence should be addressed to Dr. Mark G. Baxter, Department of Neuroscience, Mount Sinai School of Medicine, One Gustave L Levy Place, Box 1065, New York, NY 10029.

DOI:10.1523/JNEUROSCI.2947-12.2012

Copyright $\odot 2012$ the authors $\quad 0270-6474 / 12 / 3213787-09 \$ 15.00 / 0$ cluding perirhinal and entorhinal cortex (Browning et al., 2005; Davachi, 2006); (2) the hippocampus (Vargha-Khadem et al., 1997); (3) the fornix (Gaffan, 1994; Aggleton et al., 2000); (4) the mammillary bodies (Parker and Gaffan, 1997); (5) the mediodorsal nucleus of the thalamus (Gaffan and Parker, 2000); and (6) regions of the prefrontal cortex (Ranganath et al., 2003; Browning and Gaffan, 2008).

In our laboratory, we measured episodic memory in nonhuman primates using an object-in-place scene-learning task. This task shares several features particular to human episodic memory. The scenes are learned rapidly even after monkeys have experienced thousands of unique episodes, and rapid learning depends both on the unique background and the spatial and object attributes of the scene (Gaffan, 1994; Browning et al., 2005). Further, fornix transection, which leads to episodic memory impairment in humans, impairs scene learning in both humans (Aggleton et al., 2000) and monkeys (Gaffan, 1994) to identical degrees.

Past work in macaque monkeys from our laboratory has shown that selective acetylcholine depletion of the inferotemporal cortex does not impair scene learning (Browning et al., 2009). The effects of the acetylcholine depletion were only evident when combined with a structural lesion-fornix transection, which removes additional subcortical and cortical connections of the hippocampus and medial temporal lobe (Saunders and Aggleton, 2007). Fornix transection alone leads to a moderate impairment in scene learning (Gaffan, 1994; Aggleton et al., 2000), but when it is preceded by acetylcholine depletion of the inferotemporal cortex, the impairment is significantly greater-a synergistic effect (Browning et al., 2009). 
Two alternative hypotheses may explain the synergistic interaction of the acetylcholine depletion with the fornix transection. It may reflect a "double hit" to cholinergic afferents innervating the temporal lobe, because fornix transection deprives additional regions such as entorhinal cortex of cholinergic afferents (Saunders and Aggleton, 2007). Alternatively, the loss of acetylcholine in the temporal cortex may disrupt a compensatory mechanism that normally occurs after fornix transection.

Here we present evidence that acetylcholine is important for the remodeling or plasticity of the cortex following a structural lesion to part of the circuit for episodic memory. We trained macaque monkeys on object-in-place scene learning (Gaffan, 1994). We gave monkeys acetylcholine depletion of the inferotemporal cortex either before or after a structural lesion within the circuit for episodic memory (fornix transection or mammillary bodies lesion). We show that scene learning is severely impaired only when the acetylcholine depletion precedes the structural damage, regardless of the location of the structural damage. This suggests that acetylcholine depletion of inferotemporal cortex inhibits a compensatory mechanism that takes place after structural damage to the episodic memory circuitry to minimize the behavioral deficit.

\section{Materials and Methods}

\section{Subjects}

Subject were nine rhesus monkeys (Macaca mulatta; 6 male, 3 female, $2.83-6.70 \mathrm{~kg} ; 22-37$ months old at the start of behavioral testing). Monkeys were socially housed indoors in single-sex groups in enclosures attached to standard caging. The social groups included other monkeys that were involved in separate, but methodologically similar, experiments. Water was available ad libitum in the home enclosure, and each monkey's daily food ration was provided during the course of behavioral testing, or in the home enclosure on days on which testing did not take place. Data from three monkeys (all male; one rhesus and two cynomolgous, Macaca fascicularis) who received cholinergic depletions of inferotemporal cortex followed by fornix transection were taken from Browning et al. (2009) and are reported here and included in Table 1 and Figures 3 and 4 .

All of the monkeys received two lesions. One lesion was a cholinergic depletion of inferotemporal cortex and the other was either a transection of the fornix or a radiofrequency lesion of the mammillary bodies. Three monkeys received fornix transection followed by cholinergic depletion of inferotemporal cortex. Three monkeys received lesions of the mammillary bodies followed by cholinergic depletion of inferotemporal cortex. Three monkeys received cholinergic depletion of inferotemporal cortex followed by lesions of the mammillary bodies. Behavioral testing was performed following each surgery so that the effect of each surgery or combination of surgeries on memory could be assessed.

\section{Apparatus}

Behavioral testing took place in an automated apparatus in a darkened experimental cubicle. Monkeys sat in a wheeled transport cage with horizontal bars that was fixed in front of a video-display unit with a touch-sensitive screen $(380 \times 280 \mathrm{~mm}, 800 \times 600$ pixel resolution). The monkey could reach through the bars to reach the screen and the rewards. Stimulus presentation, recording of touches to the screen, and reward delivery were all under computer control. Rewards were $190 \mathrm{mg}$ banana or sugar pellets (P. J. Noyes), which were deliv-

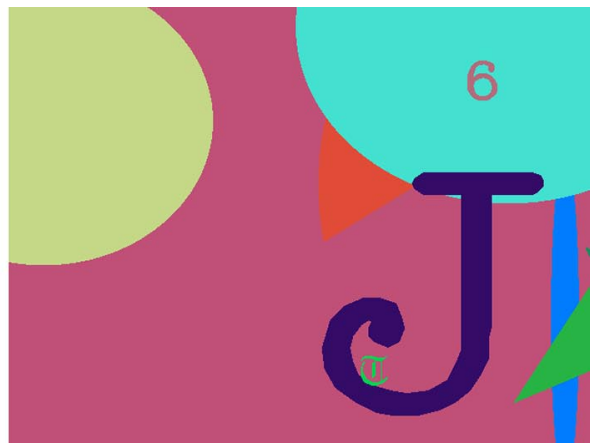

Scene-learning task. Monkeys learned new lists of scene problems in each session, in which each scene contained two ASCIl characters), of which one was consistently rewarded. The list was presented eight times per test session. earning curves reflect chance performance on the first run through each list and, preoperatively, rapid within-session learning of episodic memory.

ered into a food cup located below the touchscreen, accompanied by a click from the pellet dispenser. A metal "lunchbox" $(\sim 200 \times 100 \times$ $100 \mathrm{~mm}$ ) was located to the left of the food cup and was filled with a mixture of wet monkey chow, seeds, fruit, and nuts. The lunchbox was closed during the testing session and automatically opened following the last correct trial in the session, allowing the monkey access to a large food reward. The monkey was observed throughout with infrared cameras.

\section{Stimulus material}

The object-in-place scene learning task was adapted from Gaffan (1994). Each trial consisted of an artificially constructed scene that occupied the whole area of the display screen. Each scene was unique in five attributes, generated by an algorithm based on a random-number generator. Each scene varied in (1) the background color; (2) the location of ellipses on the screen; (3) the color, size, and orientation of ellipse elements, (4) the large alphanumeric nontarget character; and (5) the color of the character. Two of these targets, small randomly chosen colored alphanumeric characters, were placed within each scene: one correct (rewarded) and one incorrect (unrewarded) choice. These targets were fixed within and unique to each scene. All the colors were assigned with the constraint that the foreground objects should be visible (that is, there was a minimum separation in color space between the colors of a foreground object and the color of any element of its local background). Because these scenes were generated by an algorithm based on a random number generator, an infinite number of unique scenes could be generated. An example scene is shown in Figure 1.

\section{Preoperative training}

The training procedure has already been described by Browning et al. (2009). Briefly, after pretraining to enter the wheeled transport cage, take reward pellets, and eat food in the cubicle, monkeys were trained on the touchscreen. The monkeys were trained to touch colored alphanumeric characters arrayed on a black background (in a different size and typeface than those used in the scene task) and were required to touch in any location on the screen to receive a pellet. In the next phase of training, single characters were presented in random locations on the screen and remained until touched. When the character was touched, it disappeared and a pellet was delivered. The complexity of the display gradually increased on subsequent days by adding extra elements (a colored background, colored ellipse segments and a single large alphanumeric character). When monkeys were able to complete 50 trials in a single test session with minimal accuracy errors (i.e., touching locations on the screen other than the target character), they began training on the scene task.

After pretraining, monkeys were presented with a number of scenes that were repeated within the session. The session began with the presentation of a scene on the touchscreen. A touch to the correct object caused it to flash for $2.4 \mathrm{~s}$, after which a pellet was delivered and the screen blanked for an intertrial interval of 5 s. If the incorrect object was 
touched, the screen blanked for $20 \mathrm{~s}$ and the trial was repeated as a forced-choice correction trial. After the initial presentation of the scenes, they were presented seven more times in the same order as the first run through the list. In subsequent presentations, there were no correction trials. Monkeys learned which target was correct very rapidly by trial and error: in the second run through the list, error rates were low (mean = $19.53 \%$, range $=9-33 \%$; chance is $50 \%$ ). When the monkey completed the final trial of a session, the lunchbox opened and the monkey received the large food reward. If the final trial was incorrect, a correction trial was given so that the large reward was only given until after a correct response.

The number of scenes was increased gradually, based on each monkey's performance, until each monkey saw a list of between 10 and 25 scenes, depending on ability. The list was repeated at least eight times in a single session. Training continued until performance was stable. This was achieved in a mean of 16 sessions for all monkeys (range, 10-29 sessions). The dependent measure was the number of errors made after the first presentation of the list of scenes.

\section{Performance tests}

Monkeys were give 13 sessions of testing at three time points: preoperatively (Pre-op), 9-18 d after the first surgery (Post-op 1), and 12-22 d after the second surgery (Post-op 2). Before the Pre-op test, monkeys were given a break in training (12-16 d) comparable to the rest period that followed surgery. In the first two sessions of each performance, test monkeys learned fewer scenes than in the other test sessions to ensure they were motivated to work after the break in testing that followed surgery. The data from the final 10 sessions of each performance test were considered in the analysis.

\section{Surgery}

Neurosurgical procedures were performed under aseptic conditions in a dedicated operating theater. Each operated monkey received two neurosurgical procedures, separated by a period of rest and behavioral testing. The order of the two procedures (a removal of cholinergic input to inferotemporal cortex, and either a transection of the fornix or lesion of the mammillary bodies) is specified in Subjects, above. All the procedures shared some features (see below).

Steroids (methylprednisolone, $20 \mathrm{mg} / \mathrm{kg}$ ) were given intramuscularly the night before surgery and three doses were given $4-6 \mathrm{~h}$ apart (i.v. or i.m.) on the day of surgery to protect against intraoperative edema and postoperative inflammation. Each monkey was sedated on the morning of surgery with both ketamine $(10 \mathrm{mg} / \mathrm{kg})$ and xylazine $(0.25-0.5 \mathrm{mg} /$ $\mathrm{kg})$, intramuscularly. Once sedated, the monkey was given atropine $(0.05$ $\mathrm{mg} / \mathrm{kg}$ ) to reduce secretions, antibiotic (amoxicillin, $8.75 \mathrm{mg} / \mathrm{kg}$ ) for prophylaxis of infection, opioid (buprenorphine $0.01 \mathrm{mg} / \mathrm{kg}$ i.v., repeated twice at $4-6 \mathrm{~h}$ intervals on the day of surgery, i.v. or i.m.) and nonsteroidal anti-inflammatory (meloxicam, $0.2 \mathrm{mg} / \mathrm{kg}$, i.v.) agents for analgesia, and an $\mathrm{H} 2$ receptor antagonist (ranitidine, $1 \mathrm{mg} / \mathrm{kg}$, i.v.) to protect against gastric ulceration as a side effect of the combination of steroid and nonsteroidal anti-inflammatory treatment. The head was shaved and an intravenous cannula put in place for intraoperative delivery of fluids (sterile saline drip, $5 \mathrm{ml} / \mathrm{h} / \mathrm{kg}$ ). The monkey was moved into the operating theater, intubated, placed on sevoflurane $(1.5-3.75 \%$, to effect, in $100 \%$ oxygen) anesthesia, and mechanically ventilated. Some monkeys received an intravenous infusion of propofol $(10 \mathrm{mg} / \mathrm{ml})$ in addition to sevoflurane, to allow a lower concentration of sevoflurane to be used ( 3 cases, mean infusion $33.87 \mathrm{ml}$ ). An adjustable hot-air-blower blanket (Bair Hugger) allowed maintenance of normal body temperature during surgery. Heart rate, oxygen saturation of hemoglobin, mean arterial blood pressure, end tidal $\mathrm{CO}_{2}$, body temperature, and respiration rate were monitored continuously throughout surgery.

The monkey was placed in a head-holder and the head cleaned with alternating antimicrobial scrub and alcohol and draped to allow a midline or coronal incision. The skin and underlying galea were opened in layers. The temporal muscles were retracted as necessary to expose the skull surface over the intended lesion site.

When the lesion was complete, the dura was sewn over the lesion site and the bone flap replaced and held with loose sutures. Once the bone flap had been replaced, monkeys received $0.2 \mathrm{mg} / \mathrm{kg}$ metoclopramide (i.m.) to prevent postoperative vomiting. Some monkeys received $0.01 \mathrm{mg} / \mathrm{kg}$ midazolam (i.m.) to prevent seizures, if considered necessary. The galea and skin were closed in layers, and then the monkey was removed from the head-holder and anesthesia discontinued. The monkey was extubated when a swallowing reflex was evident, returned to the home cage, and monitored continuously until normal posture was regained (usually within $10 \mathrm{~min}$ ). Treatment with analgesics and antibiotics continued following surgery in consultation with veterinary staff for 3-5 d. Operated monkeys rejoined their social groups as soon as practicable after surgery, usually within $3 \mathrm{~d}$ of the operation.

Cholinergic depletion of inferotemporal cortex. All nine monkeys received bilateral injections into the inferotemporal cortex. A bone flap was turned over one temporal lobe and the craniotomy was extended with rongeurs as necessary. The dura was cut and reflected over the temporal lobe. The injection protocol is described by Browning et al. (2009): $1 \mu \mathrm{l}$ injections of $0.02 \mu \mathrm{g} / \mu \mathrm{l}$ ME20.4-saporin prepared in sterile $0.9 \%$ saline (Advanced Targeting Systems) and delivered via a Hamilton syringe guided by hand. Injections were placed first in the lateral inferotemporal cortex, each injection $\sim 3 \mathrm{~mm}$ from the others. These were aimed at the middle temporal gyrus, the inferior temporal gyrus, and both banks of the anterior middle temporal sulcus. Further injections were then made on the medial surface of the temporal lobe, in perirhinal and entorhinal cortex and the temporal pole. Once one side of the lesion was completed, the dura was sewn over the lesion site and the bone flap replaced and held with loose sutures before the second hemisphere was exposed. At the end of the procedure, the skin and galea were sewn in layers. Between 54 and 65 injections were made into the left hemisphere (mean 59.8) and between 57 and 62 in the right hemisphere (mean 59.6). The intention was to cover the equivalent extent of cortical surface to that ablated in disconnection studies of prefrontal cortex and inferotemporal cortex (Browning et al., 2005), as well as the rostral entorhinal cortex (Fig. $2 a$, gray area).

Structural lesions. Monkeys that received fornix transection underwent a protocol identical to that described by Browning et al. (2009). A bone flap was turned in a D-shape over the midline and one hemisphere. The dura was cut and reflected to expose the hemisphere up to the midline. Veins draining into the sagittal sinus were cauterized and cut. The hemisphere was retracted from the falx with a brain spoon and a glass aspirator was used to make a sagittal incision of no more than $5 \mathrm{~mm}$ in the corpus callosum at the level of the intraventricular foramen. The fornix was sectioned transversely by electrocautery and aspiration with a 20 -gauge metal aspirator that was insulated to the tip. Once the lesion was completed, the dura was replaced over the lesion site and the bone flap replaced and held with loose sutures before the skin and galea were sewn in layers.

Monkeys that received lesions of the mammillary bodies underwent a protocol identical to that described by Parker and Gaffan (1997). A bone flap was turned in a D-shape over the midline and one hemisphere. The dura was cut and reflected to expose the hemisphere up to the midline. Veins draining into the sagittal sinus were cauterized and cut. The left hemisphere was gently retracted to expose the splenium of the corpus callosum and a sagittal incision was made using a glass aspirator. The incision was made along the midline from the posterior limit of the splenium anteriorly until the tela choroidea was clearly visible over the thalamus and third ventricle. A sagittal incision was made in the tela choroidea exposing the third ventricle, massa intermedia, and dorsal surface of the thalamus. A radiofrequency thermistic probe, insulated to the tip was fixed in a Kopf stereotaxic frame at an angle of $31^{\circ}$ posterior from vertical in the sagittal plane and inserted into the third ventricle opening at the midline. X-ray pictures were used to place the probe $2 \mathrm{~mm}$ dorsal to the dorsal surface of the posterior clinoid process (Fig. $2 b$ ). The tip of the probe was heated to $80^{\circ} \mathrm{C}$ for $60 \mathrm{~s}$, except in the case of one monkey when the probe was heated to $90^{\circ} \mathrm{C}$ for $60 \mathrm{~s}$. Once the lesion was completed, the dura was replaced over the lesion site and the bone flap replaced and held with loose sutures before the skin and galea were sewn in layers. 
a
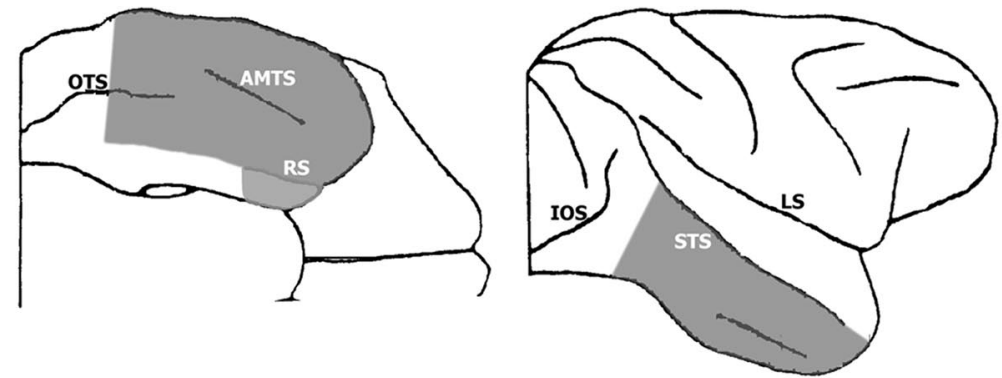

b
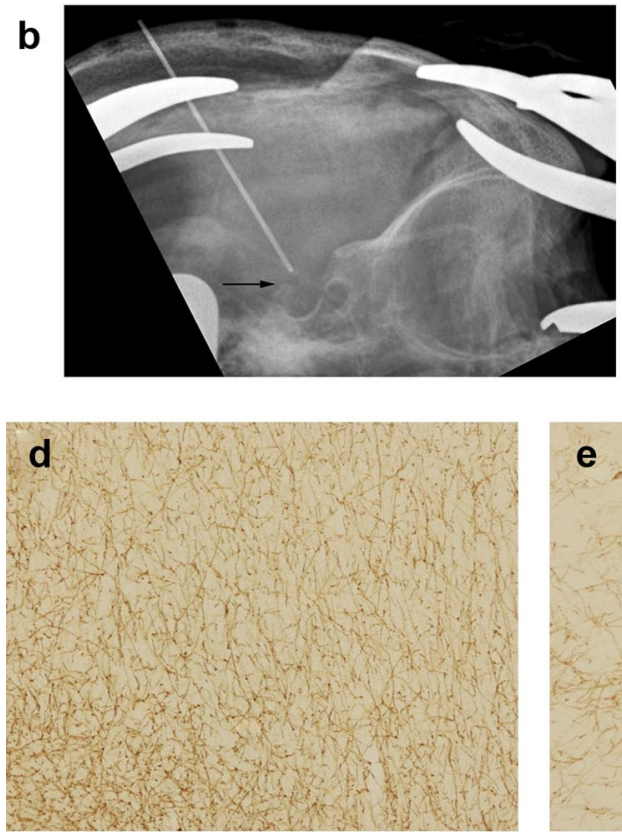

C

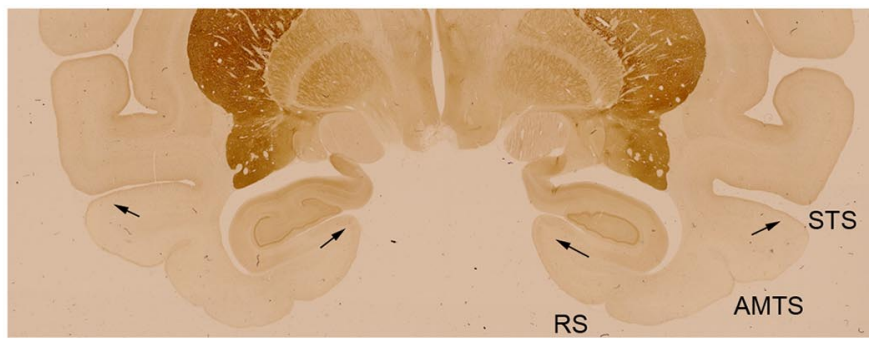

g
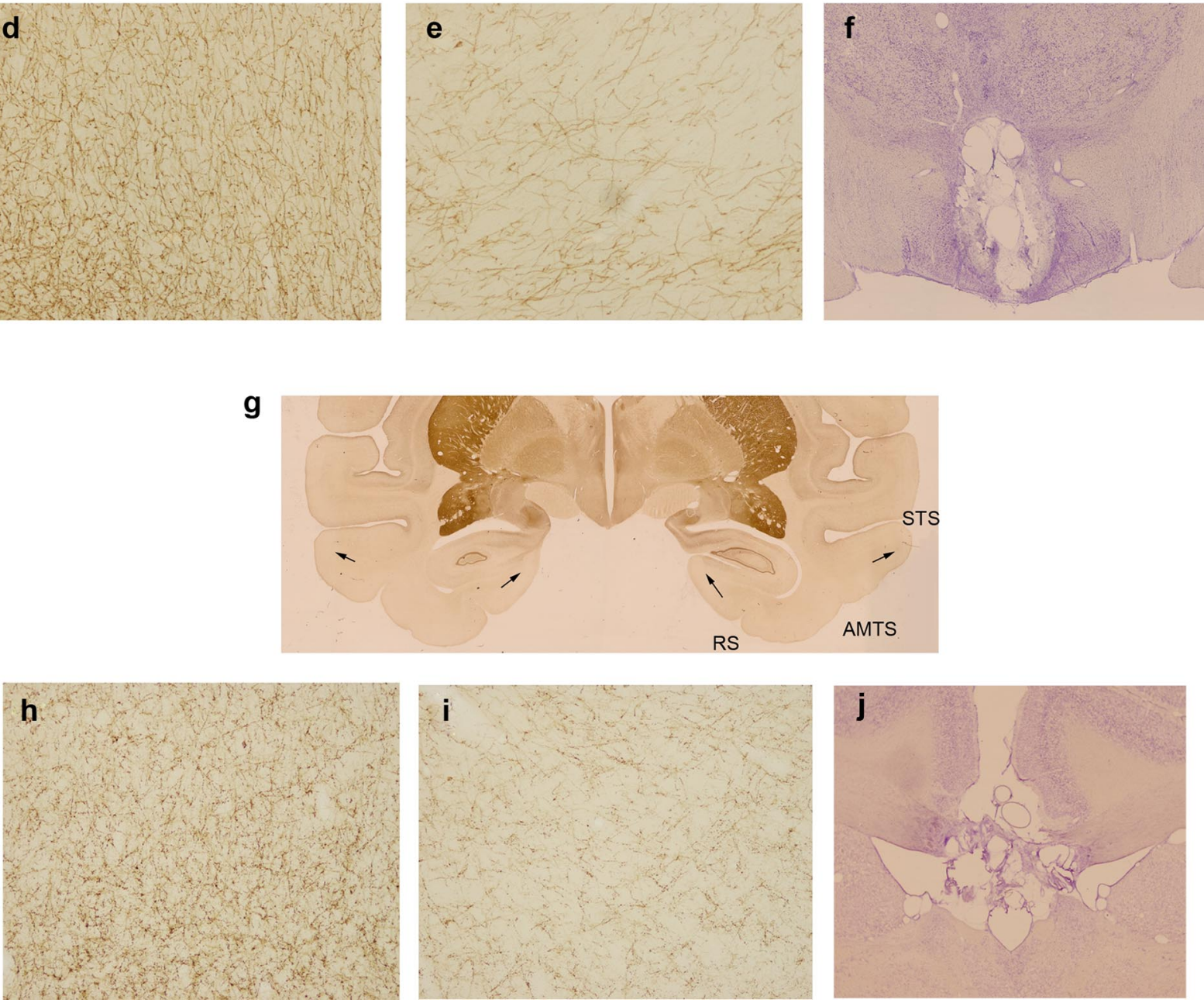

Figure 2. Lesions. $\boldsymbol{a}$, Extent of the intended depletion of cholinergic fibers in inferotemporal cortex, shown in gray. $\boldsymbol{b}-\boldsymbol{f}$, Lesion of the mammillary bodies plus inferotemporal cholinergic depletion. $\boldsymbol{g}$-j, Fornix transection plus inferotemporal cholinergic depletion. $\boldsymbol{b}$, Position of probe for lesion. $\boldsymbol{c}, \boldsymbol{g}$, Histological section stained for acetylcholinesterase. The extent of the lesions is marked with arrows. STS, Superior temporal sulcus; RS, rhinal sulcus; OTS, occipitotemporal sulcus; IOS, inferior occipital sulcus; LS, lateral sulcus. Magnification (10X) of superior temporal gyrus (control region showing dense cholinergic innervation; $\boldsymbol{d}, \boldsymbol{h}$ ) and perirhinal cortex from a reprsentative case $(\boldsymbol{e}, \boldsymbol{i}) . \boldsymbol{f}, \boldsymbol{j}$, Section taken at the level of the mammillary bodies $(\boldsymbol{f})$ or fornix $(\boldsymbol{j})$, stained with cresyl violet, illustrating the structural lesion. 
Table 1. Fiber counts expressed as a proportion of superior temporal gyrus counts in each inferior temporal cortex subregion

\begin{tabular}{|c|c|c|c|c|c|c|c|c|}
\hline \multirow[b]{2}{*}{ Case } & \multicolumn{2}{|c|}{ Entorhinal } & \multicolumn{2}{|c|}{ Perirhinal } & \multicolumn{2}{|l|}{ AMTS } & \multicolumn{2}{|l|}{ MTG } \\
\hline & Left & Right & Left & Right & Left & Right & Left & Right \\
\hline \multicolumn{9}{|c|}{ Control: Saline injection then fornix transection } \\
\hline CON1 & 0.633 & 0.682 & 1.497 & 1.190 & 1.317 & 1.401 & 1.409 & 1.154 \\
\hline CON2 & 0.603 & 0.968 & 1.000 & 1.289 & 1.089 & 1.606 & 0.828 & 0.824 \\
\hline CON3 & 0.771 & 0.801 & 1.602 & 1.244 & 1.615 & 1.724 & 1.075 & 0.959 \\
\hline \multicolumn{9}{|c|}{ ACh depletion then fornix transection } \\
\hline ACh1 & 0.653 & 0.187 & 0.626 & 0.610 & 0.441 & 0.291 & 0.140 & 0.108 \\
\hline ACh2 & 0.131 & 0.373 & 0.309 & 0.375 & 0.282 & 0.184 & 0.168 & 0.166 \\
\hline ACh3 & 0.449 & 0.333 & 0.523 & 0.439 & 0.320 & 0.215 & 0.116 & 0.122 \\
\hline Ratio to control & \multicolumn{2}{|c|}{0.49} & \multicolumn{2}{|c|}{0.37} & \multicolumn{2}{|c|}{0.20} & \multicolumn{2}{|c|}{0.13} \\
\hline \multicolumn{9}{|c|}{ Fornix transection then ACh depletion } \\
\hline Case 1 & 0.863 & 1.140 & 0.419 & 0.643 & 0.072 & 0.275 & 0.227 & 0.488 \\
\hline Case 2 & 0.183 & 0.120 & 0.105 & 0.122 & 0.140 & 0.104 & 0.008 & 0.057 \\
\hline Case 3 & 0.627 & 0.611 & 0.285 & 0.324 & 0.170 & 0.139 & 0.201 & 0.190 \\
\hline Ratio to control & \multicolumn{2}{|c|}{0.80} & \multicolumn{2}{|c|}{0.24} & \multicolumn{2}{|c|}{0.10} & \multicolumn{2}{|c|}{0.19} \\
\hline \multicolumn{9}{|c|}{ ACh depletion then mammillary bodies lesion } \\
\hline Case 4 & 2.545 & 1.649 & 1.410 & 0.255 & 0.719 & 0.092 & 0.069 & 0.011 \\
\hline Case 5 & 2.005 & 0.186 & 0.205 & 0.181 & 0.211 & 0.097 & 0.026 & 0.028 \\
\hline Case 6 & 1.155 & 2.352 & 0.107 & 0.465 & 0.153 & 0.094 & 0.013 & 0.041 \\
\hline Ratio to control & \multicolumn{2}{|c|}{2.28} & \multicolumn{2}{|c|}{0.33} & \multicolumn{2}{|c|}{0.16} & \multicolumn{2}{|c|}{0.03} \\
\hline \multicolumn{9}{|c|}{ Mammillary bodies lesion then ACh depletion } \\
\hline Case 7 & 0.865 & 2.121 & 0.719 & 0.567 & 0.221 & 0.196 & 0.176 & 0.455 \\
\hline Case 8 & 0.636 & 0.396 & 0.438 & 0.094 & 0.095 & 3.731 & 0.027 & 0.018 \\
\hline Case 9 & 1.138 & 1.845 & 0.238 & 0.193 & 0.127 & 0.064 & 0.020 & 0.021 \\
\hline Ratio to control & \multicolumn{2}{|c|}{1.55} & \multicolumn{2}{|c|}{0.28} & \multicolumn{2}{|c|}{0.48} & \multicolumn{2}{|c|}{0.12} \\
\hline Loss in fornix groups & \multicolumn{2}{|c|}{0.64} & \multicolumn{2}{|c|}{0.31} & \multicolumn{2}{|c|}{0.15} & \multicolumn{2}{|c|}{0.16} \\
\hline Loss \% & & & & & & & & \\
\hline Loss in mammillary groups & & & & & & & & \\
\hline Loss $\%$ & & & & & & & & \\
\hline
\end{tabular}

Mean depletions for each lesioned group of monkeys expressed as a ratio of the control fiber counts are shown in the "Ratio to control" row for each. Because the control monkeys had transection of the fornix, reducing cholinergic innervation of the entorhinal cortex, the counts in entorhinal cortex of monkeys with lesions of the mammillary bodies (whose fornices were intact) increased. Data from the first two groups of monkeys are taken from Browning et al. (2009) and those cases are designated as in that paper (CON1-3 and ACh1-3). Cases from the present study are designated 1 to 9.

Histology

After completion of all behavioral testing, subjects were deeply anesthetized with intravenous barbiturate and transcardially perfused with $0.9 \%$ saline followed by $4 \%$ paraformaldehyde. Brains were extracted and postfixed in $4 \%$ paraformaldehyde overnight, then cryoprotected in $30 \%$ sucrose solution. The brains were cut on a freezing microtome in $50 \mu \mathrm{m}$ sections in the coronal plane. A series of 1:10 sections was stained for acetylcholinesterase to visualize cholinergic fibers (Fig. $2 c-e, g-i$ ). The protocol for acetylcholinesterase staining is described by Browning et al. (2009). A 1:10 series of sections (adjacent to those stained for acetylcholinesterase) was stained with cresyl violet for cell bodies (Fig. $2 f ; j$ ).

To stain for GABAergic interneurons, monoaminergic fibers, and serotonergic fibers, 1:30 sections were immmunohistochemically processed for parvalbumin, tyrosine hydroxylase, and 5,7-dihydroxytryptamine transporter, respectively. Sections were incubated in the appropriate primary antibody [rabbit anti-parvalbumin, 1:15000 (Swant); mouse anti-tyrosine hydroxylase, 1:30,000; rabbit anti-5,7-dihydroxytryptamine transporter, 1:12,000 (both Immunostar)] for $2 \mathrm{~d}$. Sections were subsequently developed with standard avidin-biotin complex methods using biotinylated secondary antibodies and diaminobenzidine, with or without nickel intensification. As in previous studies using these toxins (Browning et al., 2009; Croxson et al., 2011), these stains showed no loss of dopaminergic, noradrenergic, or serotonin fibers, and spared GABAergic interneurons and overall cellular structure.

The anatomical extent of the cholinergic lesions were assessed using fiber-counting using the method described by Browning et al. (2009). Either three [for entorhinal cortex, perirhinal cortex, and anterior middle temporal sulcus (AMTS)] or five [for middle temporal gyrus (MTG) and superior temporal gyrus (STG)] slides were selected from each subject, which were spaced evenly from the anterior to posterior part of the lesion. The placement of regions of interest were as follows: the crown of the medial bank of the rhinal sulcus (entorhinal cortex), the lateral bank of the rhinal sulcus (perirhinal cortex), the medial bank of the AMTS, the superior part of MTG, and the inferior part of STG. The sections were chosen to be equally spaced (1.5-2 $\mathrm{mm}$ apart) throughout the anterior-posterior extent of the largest cortical regions (the MTG and STG). The entorhinal cortex and perirhinal cortex only extended through three of these sections, so counts from only three sections were taken; to get representative counts for the entire MTG and STG, we included three sections. For all regions, counts were averaged across the anterior-posterior images.

We analyzed fiber counts from entorhinal cortex, perirhinal cortex, AMTS, and MTG, each normalized to counts in STG (which was used as a control region) to account for variation in histological staining between monkeys. We also included fiber counts from the three control monkeys (cases CON1-CON3) in Browning et al. (2009), who received saline injections into inferotemporal cortex followed by fornix transection, for comparison. There was no significant difference between raw counts in the STG when comparing controls and monkeys that had received a cholinergic depletion $\left(F_{(1,13)}=1.053, p=0.323\right)$ or comparing monkeys that had received fornix transection and mammillary bodies lesion $\left(F_{(1,10)}=1.988, p=0.189\right)$, supporting the use of the STG as a control region.

The fiber counts for each region were expressed as a proportion of counts in the STG (Table 1). Analysis of the fiber depletion in a repeated-measures ANOVA with within-subjects factors of region (4 levels: entorhinal, perirhinal, AMTS, MTG) and hemisphere (2 levels) and between-subjects factor of group (5 levels) showed that there was a main effect of group $\left(F_{(4,10)}=12.974, p=0.001\right)$. There was also a main effect of region $\left(F_{(4,40)}=12.885, p<0.001\right)$ and an interaction between group and region $\left(F_{(16,40)}=4.441, p=0.002\right)$. There was no main effect of hemisphere or interaction with hemisphere with any other factor $(F<2$, $p>0.1$ in all cases). Duncan's test performed post hoc revealed that the controls alone differed from the other groups $(p<0.05)$, while the other four groups were homogeneous. 
Critically, three further comparisons were performed using a repeated-measures ANOVA; between the two groups that had received fornix transections and the two groups that had received lesions of the mammillary bodies. For the fornix groups, there was a main effect of region $\left(F_{(4,16)}=39.042, p<0.001\right)$ but no main effect of group $\left(F_{(1,4)}<0.001, p=0.988\right)$ and no interaction between group and region $\left(F_{(4,16)}=\right.$ $2.341, p=0.160)$. For the mammillary bodies groups, there was also a main effect of region $\left(F_{(4,16)}=8.307, p=0.010\right)$ but no main effect of $\operatorname{group}\left(F_{(1,4)}=0.008, p=0.934\right)$ and no interaction between group and region $\left(F_{(4,16)}=0.943\right.$, $p=0.431)$. A repeated-measures ANOVA including just the fornix groups and the mammillary bodies groups revealed no main effect of group $\left(F_{(3,8)}=2.595, p=0.125\right)$, a main effect of region $\left(F_{(3,24)}=10.530, p<0.001\right)$, and an interaction between group and region $\left(F_{(9,24)}=2.302\right.$, $p=0.050)$. Thus, the cholinergic lesions were equivalent in extent between monkeys that received the lesion as the first or second surgical procedure, and in monkeys whose structural lesion was of the fornix or mammillary bodies. There was almost no apparent loss of entorhinal cortex fibers in monkeys with a mammillary lesion compared with controls. The apparent elevation of entorhinal fiber counts in monkeys with mammillary lesions is caused by comparatively low fiber counts in the entorhinal cortex of control sections, which were taken from monkeys in Browning et al. (2009) that had received fornix transection, depriving the entorhinal cortex of many of its cholinergic inputs. In the other cortical areas, there was extensive depletion, comparable to the other groups.

\section{Results}

As in monkeys with fornix transection (Fig. 3a), there was a synergistic effect of combined mammillary body lesions and inferior temporal cholinergic depletion (Fig. $3 b$ ). The initial inferior temporal cholinergic depletion caused no deficit, but subsequent lesion of the mammillary bodies produced a larger deficit than would be expected following a mammillary body lesion alone. We compared the fornix and mammillary body structural lesion groups with a repeated-measures ANOVA with group (2 levels) as the between-subjects factor and operative stage (3 levels) and trial (8 levels) as the within-subjects factors. There was no significant main effect of group $\left(F_{(1,4)}<0.001, p=0.988\right)$ or interaction between group and operative stage $\left(F_{(2,8)}=1.120, p=\right.$ 0.373 ), indicating similar patterns of effects between the two different structural lesions. Both groups showed a deficit after the structural lesion, indicated by a significant main effect of operative stage $\left(F_{(2,8)}=91.996, p<0.001\right)$. Thus, loss of temporal cortical acetylcholine exacerbates the amnestic effects of different structural lesions upstream within the neural circuitry for episodic memory.

To test the double-hit explanation of these results, we compared these data to those of monkeys who also had combined lesions of either the fornix or mammillary bodies and the temporal cortical cholinergic projections, but who sustained the structural lesion before the cholinergic lesion. In both



d

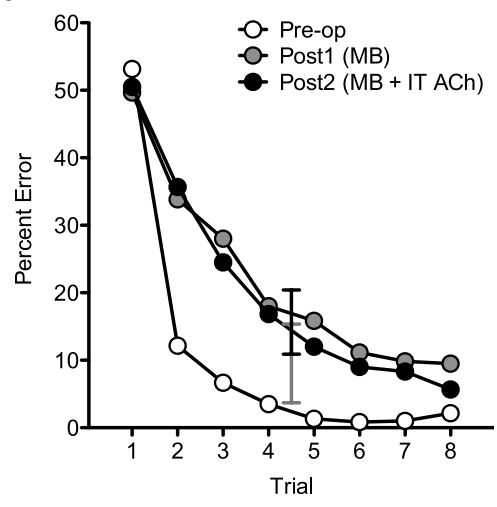

Figure 3. Scene learning performance. Each data point represents the mean for monkeys in each group at each phase of testing Pre-op, Post1, Post2). The free-floating error bars represent twice the standard error of the difference in means across all eight phases within each group, as recommended by Cardinal and Aitken (2006). The gray error bars represent the difference between ost 1 , and the black error bars represent the difference between Post1 and Post2. $\boldsymbol{a}$, Results reproduced from Browning the addition of a fornix transection they were severely impaired. $\boldsymbol{b}$, The identical pattern of data was observed in monkeys that . magnitude to monkeys receiving fornix transection after inferotemporal cholinergic depletion. $\boldsymbol{c}, \boldsymbol{d}$, When the order of the lesions was reversed, lesions of the fornix (c) or mammillary bodies $(\boldsymbol{d})$ caused significant but mild impairment, which was not exacerbated by subsequent inferotemporal cholinergic depletion.

cases, monkeys were mildly impaired at the scene learning task following the structural lesion, as expected, but the impairment was not exacerbated by subsequent inferior temporal cholinergic depletion (Fig. $3 c, d$ ). We compared the effect of lesion order with a repeated-measures ANOVA with order (2 levels: cholinergic depletion first or second) and structural lesion identity (2 levels: fornix or mammillary bodies) as between-subjects factors and operative stage (3 levels) and trial (8 levels) as within-subjects factors. There was no overall main effect of order $\left(F_{(1,8)}=0.133, p=0.725\right)$, but there was a significant interaction between operative stage and order $\left(F_{(2,16)}=39.651, p<0.001\right)$ and a three-way interaction between operative stage, order, and trial $\left(F_{(14,112)}=10.443, p<\right.$ $0.001)$. These interactions indicate that the combination of lesions produces a different effect depending on the order in which the lesions occur. There was no main effect of structural lesion identity and no significant interactions between operative stage and structural lesion $(p>0.05)$. Summary data for individual subjects are listed in Table 2. Histological analyses indicated that cholinergic lesion extent was comparable in all experimental groups (Table 1), so the different effects of lesion 
Table 2. Individual subject data in scene learning for monkeys illustrated in Figure 3

\begin{tabular}{lccc}
\hline Case & Pre-op & Post1 & Post2 \\
\hline ACh depletion then fornix transection & & & \\
ACh1 & 3.26 & 2.97 & 29.2 \\
ACh2 & 12.71 & 16.14 & 37.14 \\
ACh3 & 9.5 & 8 & 30.07 \\
$\quad$ Mean & 8.49 & 9.04 & 32.14 \\
Fornix transection then ACh depletion & & & \\
$\quad$ Case 1 & 3.29 & 12.57 & 7.86 \\
Case 2 & 11.93 & 33.79 & 23.86 \\
Case 3 & 13.43 & 28.71 & 25 \\
Mean & 9.55 & 25.02 & 18.91 \\
ACh depletion then mammillary bodies lesion & & & \\
$\quad$ Case 4 & 3.57 & 5.14 & 29.86 \\
Case 5 & 5.07 & 7.21 & 26.21 \\
Case 6 & 9.86 & 23.71 & 42.14 \\
Mean & 6.17 & 12.02 & 32.74 \\
Mammillary bodies lesion then ACh depletion & & & \\
$\quad$ Case 7 & 2.64 & 22.5 & 14.79 \\
Case 8 & 7.07 & 20.43 & 20.93 \\
Case 9 & 2.14 & 11.14 & 12.29 \\
Mean & 3.95 & 18.02 & 16.00 \\
\hline
\end{tabular}

Each value represents the mean errors in trials $2-8$ of scene learning for each phase (Pre-op, Post1, Post2) for each case. Data from the first group of monkeys are taken from Browning et al. (2009) and those cases are designated as in that paper (ACh1-3). Cases from the present study are designated 1 to 9 , as in Table 1.

order cannot be explained by (for example) more extensive cholinergic lesions in the monkeys who received this as their first surgical procedure. Thus, the more severe amnesia in monkeys that receive a fornix or mammillary lesion after loss of temporal cortical acetylcholine cannot be explained by a double-hit additive effect or unmasking of the effect of a subthreshold lesion effect on behavior. This order effect also implies that the involvement of acetylcholine in supporting recovery from structural damage is transient, such that once behavioral recovery following structural damage has taken place, acetylcholine is not necessary to maintain it. In Figure 4, we show the results of this study and Browning et al. (2009) expressed as a percentage of the maximum possible deficit on the scene-learning task (chance performance on this task is $50 \%$ ), facilitating comparison of the magnitude of impairment in the different lesion conditions. This emphasizes the similarity of impairment after single lesions of the fornix or mammillary bodies with the impairment caused by these lesions when followed by cholinergic depletion, and the more severe impairment (approximately by a factor of 2 by this measure) caused by fornix or mammillary lesions preceded by cholinergic depletion.

\section{Discussion}

We have shown that the combination of a structural brain lesion with a selective neurochemical lesion has different effects on memory depending on the order in which the lesions were sustained. Monkeys that received a cholinergic depletion of inferotemporal cortex followed by a structural lesion (fornix or mammillary bodies) were severely impaired at a scenelearning task. However, monkeys that received the structural lesions first and the cholinergic depletion second were only mildly impaired. Our results indicate that the presence of acetylcholine in the temporal cortex facilitates functional recovery after damage to the fornix or mammillary bodies.

We did not see a deficit in scene learning following the cholinergic depletion of inferotemporal cortex on its own.

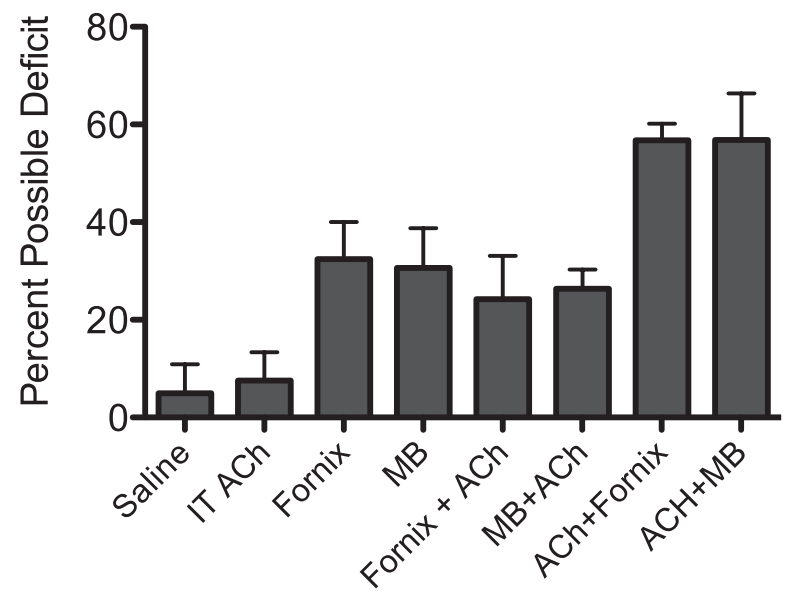

Figure 4. Comparison of each lesion type. Mean errors made in trials 2-8, expressed as a percentage of the maximum possible deficit, i.e. (postoperative - preoperative errors)/ (chance - preoperative errors), and grouped by lesion type. Monkeys that received a cholinergic depletion before the structural lesion were severely impaired at scene learning, compared with a mild impairment following a structural lesion and no impairment with cholinergic depletion alone. $x$-axis labels are as follows: Saline, saline vehicle injections into inferotemporal cortex; IT ACh, inferotemporal cholinergic depletion; Fornix, fornix transection; MB, mammillary body lesion. For combined lesions, the order of lesions is shown.

This was initially surprising, as the cholinergic system has been proposed to have an important role in learning, for example by increasing the rate of learning via improved signal/cue detection (Kilgard et al., 2002; Parikh et al., 2007). One possibility is that the involvement of acetylcholine in cue detection is mediated by prefrontal or medial frontal cortex (Kilgard et al., 2002; Parikh et al., 2007) rather than inferotemporal cortex. However, we recently showed that extensive cholinergic depletions of lateral and orbital prefrontal cortex also did not impair scene learning (Croxson et al., 2011). Cholinergic modulation of cue detection may not be critical to efficient memory in this task, perhaps because of the extensive familiarity of the monkeys with the requirements for locating cues in the complex scenes, and the lack of any temporal constraints on cue detection or responding.

We show here that although cholinergic inputs to inferotemporal cortex are not essential for rapid acquisition of episodic memories, they do modulate the effects of structural lesions of the fornix or mammillary bodies, both part of the circuit for episodic memory. This appears to be an effect on recovery of function following structural damage, because the synergistic effect of the lesions is seen only when the cholinergic lesion precedes the structural lesion, not when it follows it. This implies that normally after structural damage, a cholinergic-dependent process in inferotemporal cortex supports partial recovery of episodic memory function, and once this recovery has occurred, acetylcholine is not necessary to maintain it. This also confirms that rather than this effect being due to damage to more than one component of multiple, possibly redundant routes, it is specific to situations in which cholinergic depletion precedes the structural damage. Discerning a potential cellular or molecular mechanism for this is not straightforward because of the complex nature of the task.

A number of studies in rodents have addressed the role of the cholinergic system in plasticity. In primary sensory systems, cortical map reorganization is a potentially critical feature of learning. The reorganization of these maps, which is dependent on 
cholinergic inputs from the nucleus basalis, occurs during the first days of motor learning (Conner et al., 2003). In the auditory system the cholinergic inputs play a critical role in a form of associative learning: the remodeling of receptive fields in response to auditory stimulation (Kilgard and Merzenich, 1998; Weinberger, 2003). The role of cortical map organization is also thought to be transient- - several days after reorganization the map then renormalizes back to its original configuration, leaving learning intact (Molina-Luna et al., 2008; Reed et al., 2011). There is also evidence from primary sensory systems that reorganization after injury uses similar cholinergic-mediated mechanisms to learning. In the motor system, acetylcholine mediates cortical plasticity associated with recovery of function following motor cortex damage (Conner et al., 2005). A mechanism similar to this could explain the time-sensitive nature of the cholinergic deficit in our study. In other words, cholinergic depletions of inferotemporal cortex only affected scene learning when they occurred before the structural lesion because the cholinergic system is required for plasticity to develop, but not to be maintained. Our results suggest that these phenomena also occur in higherorder cortical association areas beyond primary sensory and motor cortex, and impact the functional organization of cognitive abilities as well. They are consistent with findings in rats that basal forebrain lesions and structural damage to entorhinal cortex (Traissard et al., 2007) or hippocampus (Craig et al., 2008, 2009) have synergistic effects on memory and, critically, suggest a potential mechanism for the synergism by showing that these effects depend on the order in which the injuries occur.

The mechanism of lasting cortical plasticity in the inferotemporal cortex could occur in a way similar to the mechanism proposed for auditory associative learning. It has been suggested that at the time of initial learning, new dendritic spines will form, which are subsequently reduced to a small population capable of maintaining behavior in the long-term (Yang and Maunsell, 2004; Xu et al., 2009).

Our findings suggest a new explanation for the functional significance of loss of basal forebrain cholinergic neurons in patients with Alzheimer's disease. The current findings could also explain some of the effects of procholinergic drug therapy in Alzheimer's disease. If the ability of the cortex to reorganize itself in response to structural damage is impaired by the loss of acetylcholine, it would explain why procholinergic drugs may slow the rate of cognitive decline in Alzheimer's disease (Farlow et al., 2005; Small et al., 2005), as they restore the capacity of the cortex to adapt to ongoing structural damage in the form of cortical neurodegeneration. Although this may relate to direct effects of particular compounds on, for example, A $\beta$ deposition (Kihara et al., 1997), it may also reflect cortical adaptation to the ongoing loss of neurons caused by Alzheimer's disease pathology (Mesulam, 2004; Craig et al., 2011).

A better understanding of the involvement of acetylcholine in these adaptive/plastic processes may lead both to a more complete understanding of the pathophysiology of cognitive impairments in dementia and amnesia, as well as to novel therapies for these conditions.

\section{References}

Aggleton JP, McMackin D, Carpenter K, Hornak J, Kapur N, Halpin S, Wiles CM, Kamel H, Brennan P, Carton S, Gaffan D (2000) Differential cognitive effects of colloid cysts in the third ventricle that spare or compromise the fornix. Brain 123:800-815. CrossRef Medline

Bartus RT (2000) On neurodegenerative diseases, models, and treatment strategies: lessons learned and lessons forgotten a generation following the cholinergic hypothesis. Exp Neurol 163:495-529. CrossRef Medline

Browning PG, Gaffan D (2008) Global retrograde amnesia but selective anterograde amnesia after frontal-temporal disconnection in monkeys. Neuropsychologia 46:2494-2502. CrossRef Medline

Browning PG, Easton A, Buckley MJ, Gaffan D (2005) The role of prefrontal cortex in object-in-place learning in monkeys. Eur J Neurosci 22:32813291. CrossRef Medline

Browning PG, Gaffan D, Croxson PL, Baxter MG (2009) Severe scene learning impairment, but intact recognition memory, after cholinergic depletion of inferotemporal cortex followed by fornix transection. Cereb Cortex 20:282-293. CrossRef Medline

Cardinal RN, Aitken MR (2006) ANOVA for the behavioral sciences researcher. Hillsdale, NJ: Lawrence Erlbaum Associates.

Conner JM, Culberson A, Packowski C, Chiba AA, Tuszynski MH (2003) Lesions of the basal forebrain cholinergic system impair task acquisition and abolish cortical plasticity associated with motor skill learning. Neuron 38:819-829. CrossRef Medline

Conner JM, Chiba AA, Tuszynski MH (2005) The basal forebrain cholinergic system is essential for cortical plasticity and functional recovery following brain injury. Neuron 46:173-179. CrossRef Medline

Craig LA, Hong NS, Kopp J, McDonald RJ (2008) Reduced cholinergic status in hippocampus produces spatial memory deficits when combined with kainic acid induced seizures. Hippocampus 18:1112-1121. CrossRef Medline

Craig LA, Hong NS, Kopp J, McDonald RJ (2009) Selective lesion of medial septal cholinergic neurons followed by a mini-stroke impairs spatial learning in rats. Exp Brain Res 193:29-42. CrossRef Medline

Craig LA, Hong NS, McDonald RJ (2011) Revisiting the cholinergic hypothesis in the development of Alzheimer's disease. Neurosci Biobehav Rev 35:1397-1409. CrossRef Medline

Croxson PL, Kyriazis DA, Baxter MG (2011) Cholinergic modulation of a specific memory function of prefrontal cortex. Nat Neurosci 14:1510 1512. CrossRef Medline

Davachi L (2006) Item, context and relational episodic encoding in humans. Curr Opin Neurobiol 16:693-700. CrossRef Medline

Farlow MR, Small GW, Quarg P, Krause A (2005) Efficacy of rivastigmine in Alzheimer's disease patients with rapid disease progression: results of a meta-analysis. Dement Geriatr Cogn Disord 20:192-197. CrossRef Medline

Gaffan D (1994) Scene-specific memory for objects: a model of episodic memory impairment in monkeys with fornix transection. J Cogn Neurosci 6:305-320. CrossRef

Gaffan D, Parker A (2000) Mediodorsal thalamic function in scene memory in rhesus monkeys. Brain 123:816-827. CrossRef Medline

Kihara T, Shimohama S, Sawada H, Kimura J, Kume T, Kochiyama H, Maeda T, Akaike A (1997) Nicotinic receptor stimulation protects neurons against beta-amyloid toxicity. Ann Neurol 42:159-163. CrossRef Medline

Kilgard MP, Merzenich MM (1998) Cortical map reorganization enabled by nucleus basalis activity. Science 279:1714-1718. CrossRef Medline

Kilgard MP, Pandya PK, Engineer ND, Moucha R (2002) Cortical network reorganization guided by sensory input features. Biol Cybern 87:333-343. CrossRef Medline

Mesulam M (2004) The cholinergic lesion of Alzheimer's disease: pivotal factor or side show? Learn Mem 11:43-49. CrossRef Medline

Molina-Luna K, Hertler B, Buitrago MM, Luft AR (2008) Motor learning transiently changes cortical somatotopy. Neuroimage 40:1748-1754. CrossRef Medline

Parikh V, Sarter M (2008) Cholinergic mediation of attention: contributions of phasic and tonic increases in prefrontal cholinergic activity. Ann N Y Acad Sci 1129:225-235. CrossRef Medline

Parikh V, Kozak R, Martinez V, Sarter M (2007) Prefrontal acetylcholine release controls cue detection on multiple timescales. Neuron 56:141154. CrossRef Medline

Parker A, Gaffan D (1997) Mamillary bodies lesions in monkeys impair object-in-place memory: functional unity of the fornix-mamillary system. J Cogn Neurosci 9:512-521. CrossRef

Ranganath C, Johnson MK, D’Esposito M (2003) Prefrontal activity associated with working memory and episodic long-term memory. Neuropsychologia 41:378-389. CrossRef Medline

Reed A, Riley J, Carraway R, Carrasco A, Perez C, Jakkamsetti V, Kilgard MP 
(2011) Cortical map plasticity improves learning but is not necessary for improved performance. Neuron 70:121-131. CrossRef Medline

Sahakian BJ, Owen AM, Morant NJ, Eagger SA, Boddington S, Crayton L, Crockford HA, Crooks M, Hill K, Levy R (1993) Further analysis of the cognitive effects of tetrahydroaminoacridine (THA) in Alzheimer's disease: assessment of attentional and mnemonic function using CANTAB. Psychopharmacology (Berl) 110:395-401. CrossRef Medline

Saunders RC, Aggleton JP (2007) Origin and topography of fibers contributing to the fornix in macaque monkeys. Hippocampus 17:396411. CrossRef Medline

Small GW, Kaufer D, Mendiondo MS, Quarg P, Spiegel R (2005) Cognitive performance in Alzheimer's disease patients receiving rivastigmine for up to 5 years. Int J Clin Pract 59:473-477. CrossRef Medline

Traissard N, Herbeaux K, Cosquer B, Jeltsch H, Ferry B, Galani R, Pernon A, Majchrzak M, Cassel JC (2007) Combined damage to entorhinal cortex and cholinergic basal forebrain neurons, two early neurodegen- erative features accompanying Alzheimer's disease: effects on locomotor activity and memory functions in rats. Neuropsychopharmacology 32:851-871. CrossRef Medline

Vargha-Khadem F, Gadian DG, Watkins KE, Connelly A, Van Paesschen W, Mishkin M (1997) Differential effects of early hippocampal pathology on episodic and semantic memory. Science 277:376-380. CrossRef Medline

Weinberger NM (2003) The nucleus basalis and memory codes: auditory cortical plasticity and the induction of specific, associative behavioral memory. Neurobiol Learn Mem 80:268-284. CrossRef Medline

Xu T, Yu X, Perlik AJ, Tobin WF, Zweig JA, Tennant K, Jones T, Zuo Y (2009) Rapid formation and selective stabilization of synapses for enduring motor memories. Nature 462:915-919. CrossRef Medline

Yang T, Maunsell JH (2004) The effect of perceptual learning on neuronal responses in monkey visual area V4. J Neurosci 24:1617-1626. CrossRef Medline 